\title{
Preliminary Support for Group Cognitive Behavioral Therapy (CBT) to Reduce Psychological Distress in Patients with Spontaneous Coronary Artery Dissection (SCAD)
}

\author{
Kelsey C. Vaca ${ }^{1}$. Jennifer A. Tremmel ${ }^{2} \cdot$ Katharine S. Edwards ${ }^{2}$ (I) \\ Accepted: 26 June 2021 / Published online: 9 July 2021 \\ (c) The Author(s), under exclusive licence to Springer Science+Business Media, LLC, part of Springer Nature 2021
}

\begin{abstract}
Spontaneous coronary artery dissection (SCAD) can occur as an atypical cause of myocardial infarction. Preliminary evidence suggests that SCAD patients experience high rates of post-event psychological distress. It is unknown whether psychosocial interventions may reduce the distress burden. Seven SCAD patients (mean age $=53.3$ years) completed a CBT-based support group. All seven participants completed measures for anxiety, depression, and cardiac-related quality of life at baseline and post-intervention, and five participants completed measures at 3-month follow-up. Six of 7 participants scored above the clinical threshold on a measure of anxiety at baseline and posttreatment. At follow-up, 3 of 5 participants scored below the clinical threshold. For depression, 3 of 7 reported elevated depressive symptoms at baseline. By follow-up, 1 of 5 endorsed elevated depressive symptoms. This is the first known psychosocial intervention study of patients with SCAD. Anxiety symptoms improved for most patients by follow-up with some patients having improved depressive symptoms. Although the sample size is limited, this pilot study suggests a potential benefit of group psychosocial interventions for SCAD survivors.
\end{abstract}

Keywords Spontaneous coronary artery dissection $\cdot$ Group psychotherapy $\cdot$ Anxiety $\cdot$ Depression

\section{Introduction}

SCAD is a unique cardiac event that disproportionately affects young women and often results in myocardial infarction (MI) (Kim, 2020). SCAD is caused by a tear in the coronary artery rather than an atherosclerotic plaque and may lead to death (Hayes et al., 2018). It is believed to be the cause of up to $4 \%$ of all acute coronary syndromes (ACS) and up to $35 \%$ of MIs that occur in women under the age of 50 (Hayes et al., 2018). The causes of SCAD are largely unknown, although it has been associated with underlying collagen vascular disorders, inflammatory diseases, hormonal influences, and genetic factors, and can be precipitated by emotional or physical stressors. At present, there are no known treatments for SCAD aside from beta

Katharine S. Edwards

ksedwards@stanford.edu

1 PGSP-Stanford Psy.D. Consortium, Palo Alto University, Palo Alto, CA, USA

2 Division of Cardiovascular Medicine, Stanford University School of Medicine, Stanford, CA 94305, USA blockers, which may reduce the risk of recurrence (Saw et al., 2017). SCAD recurrence is frequently associated with major adverse cardiac events such as recurrent MI, unanticipated revascularization, congestive heart failure, stroke, and sometimes death (Hayes et al., 2018). Recurrence of MI, primarily due to SCAD, occurs in about $17-18 \%$ of patients 3-4 years following an initial SCAD event (Kim, 2020).

Patients who have experienced SCAD are often emotionally distressed. Recent studies suggest that female patients experience their SCAD event as highly stressful and report ongoing stress about their heart health. Patients used words such as "scary," "surprising," "life changing," and "traumatic" to describe their SCAD experience (Wagers et al., 2018 , p. 3). A cross-sectional study of 158 SCAD survivors found that $33 \%$ of patients had received medication or counseling for depression, and 37\% for anxiety, in the mean 3.7 years since their initial SCAD diagnosis (Liang et al., 2014). A recent study ( $n=512$ ) of SCAD survivors, who completed the survey an average of 4.3 years after receiving their SCAD diagnosis, found that $41 \%$ of survivors indicated at least mild anxiety symptoms and $32 \%$ reported at least mild depressive symptoms, with $16 \%$ and $15 \%$ of the sample 
having scores suggesting a clinical diagnosis of anxiety and depression, respectively (Johnson et al., 2020).

Psychosocial group interventions have been successful in reducing distress and mortality in men and women with coronary artery disease who have experienced ACS. Blumenthal et al. (2005) demonstrated that a 16-week stress management training program reduced emotional distress and improved cardiovascular risk markers for patients in cardiovascular rehabilitation, at posttreatment, compared with those who did exercise-only rehabilitation or usual care. They later extended these findings to show that patients who completed the training had lower rates of clinical cardiac events at a median of 3.2 years follow-up (Blumenthal et al., 2016). Orth-Gomér et al. (2009) found that a 20-session education, stress management, and support group for female cardiac patients was associated with a significant reduction in all-cause mortality at a mean of 7.1 years follow-up compared with those who had received usual care. Both of these group interventions included components of medical education, skills training in cognitive and behavioral stress reduction strategies, and social support.

Although mental health treatment is recommended for SCAD patients with elevated anxiety and depression (Kim, 2020), there is little existing research on psychosocial group interventions for female SCAD patients. One study described a 6-month cardiac rehabilitation program designed specifically for SCAD survivors, which incorporated psychoeducation, counseling, mindful living sessions, and peer support. The details of the intervention are not described though it occurred in the context of regular exercise sessions, resistance training, and case management with referrals to social work and psychiatry as needed (Chou et al., 2016). The aim of the current pilot study was to investigate the feasibility and effectiveness of an 8-week CBT-based psychosocial support group for SCAD survivors.

\section{Methods}

Current patients identified by their cardiologists as having had a SCAD event $(n=21)$ were sent letters inviting them to participate in a psychosocial group for SCAD survivors. Female patients over the age of 18 with a diagnosis of SCAD were eligible. Individuals with acute mental health problems, significant cognitive impairment, or those who were unable to read, write, or understand English were excluded. Eligible patients were provided with informed consent to participate in the study and completed psychosocial questionnaires at baseline before the first session, at posttreatment after the last session, and at 3-month follow-up by mail. Paper-and-pencil questionnaires asked about demographic variables, date(s) of SCAD event(s), history of mental health treatment, psychosocial status, and included measures of cardiac-related quality of life, depression, and anxiety. This study was reviewed and approved by the Stanford University Institutional Review Board.

The treatment consisted of weekly 2 -h face-to-face group sessions for eight consecutive weeks. Each session began with a relaxation or mindfulness exercise $(15 \mathrm{~min})$, individual check-ins (20 min), a didactic discussion (30 min), break (10 min), and group discussion (45 min). The group intervention was CBT-based and included psychoeducation about SCAD, psychoeducation about stress, anxiety, and depression, behavioral stress management techniques, such as diaphragmatic breathing and progressive muscle relaxation, and cognitive restructuring techniques. The intervention also included acceptance and commitment therapy (ACT)-based acceptance and values techniques for coping with the uncertainty of the diagnosis (e.g., values card sort, committed action) and dialectical behavior therapy (DBT)based interpersonal effectiveness skills (e.g., assertiveness, boundary setting) for improving relationships with medical providers and family members. Patients were asked to complete homework assignments describing their experiences with SCAD and to practice the skills taught during group.

\section{Measures}

The State-Trait Anxiety Inventory (STAI; Speilberger \& Gorsuch, 1983) is a self-report measure with subtests to assess and distinguish between trait- and state-based anxieties. This study administered the 20 -item state anxiety form (STAI-Y1), which assesses the degree of anxiety experienced in the present moment and uses a 4-point scale to rate statements such as "I feel upset" from Not at all (1) to Very much so (4). A reliability generalizability study found the state anxiety scale has good internal consistency (mean $\alpha=0.92$ ) (Barnes et al., 2002), and higher scores among individuals during stressful circumstances provide evidence of its construct validity (Speilberger \& Gorsuch, 1983). A recent validity study of anxiety disorders in cardiac patients found $\geq 40$ as an optimal cut-off score for screening the presence of an anxiety disorder (Bunevicius et al., 2013). Although there are some suggested statistical methods for estimating clinically meaningful change, there is no widely accepted method.

The Patient Health Questionnaire (PHQ-9; Kroenke et al., 2001) is a frequently used self-report questionnaire for screening depressive symptoms or tracking their severity. Its psychometric properties are good, demonstrating good internal reliability (Cronbach's $\alpha=0.89$ in primary care samples, 0.86 in Ob-Gyn samples) (Kroenke et al., 2001) and validity in clinical studies of medical patients (Gilbody et al., 2007; Thekkumpurath et al., 2011). The PHQ-9 is widely used for assessment of depression in cardiac patients (Elderon et al., 2011; Lichtman et al., 2008) and a cut-off score of $\geq 10$ 
is recommended for patients with coronary heart disease (McManus et al., 2005). A 5-point decrease suggests that a clinically significant improvement has occurred (Kroenke, 2012; Löwe et al., 2004).

The Seattle Angina questionnaire (SAQ; Spertus et al., 1995) includes 19 items measuring 5 dimensions of functional health status in patients with coronary artery disease (CAD) and has been shown to have good psychometric properties and clinical utility. The Seattle Angina questionnaire-7 item version (SAQ-7; Chan et al., 2014) is a short-form of the original scale. It is composed of subscales assessing 3 aspects of present health status in CAD: Physical limitation, Angina frequency, and quality of life. Item scales range from 1 to 5 or 1 to 6 , but subscale scores are an unweighted average rescaled to a range of 0 (worst status) to 100 (best status). A summary score of the subscales is also calculated using the same procedure. A comparison of the SAQ-7 with the full scale SAQ yielded high concordance ranging from $(\geq 0.88$ to 1.00$)$ for the 3 subscales and the summary scale in patients with acute MI. It also demonstrated high test-retest reliability in stable patients ( $r s$ ranging from 0.78 to 0.86 for the subscale and summary scores) and high predictive validity of later hospitalization (Chan et al., 2014).

\section{Results}

Eight participants enrolled in the group, but one dropped out midway through the eight sessions due to an exacerbation of posttraumatic stress. All seven participants who completed the group were women who ranged in age from 42 to 61 years (mean $=53.3$ years). Time since their initial SCAD event varied. One participant's initial event had occurred over 11 years earlier. The rest of the participants experienced their first event from 4 to 55 months prior to the start of the group. Participants self-reported their racial identity, with 4 indicating White and the other 3 reporting Asian. Five of the women (71\%) had earned at least a bachelor's degree and 6 participants (86\%) reported an annual household income above $\$ 150,000$. Five of the $7(71 \%)$ reported a history of mental health treatment and 3 of these women considered their mental health treatment to be related to their SCAD event(s).

Data for participants' mean baseline, posttreatment, and follow-up scores on measures of anxiety, depression, and cardiac-related quality of life are in Table 1 . At baseline and at posttreatment, 6 (86\%) participants scored above a clinical threshold of elevated anxiety (STAI $\geq 40$; Bunevicius et al., 2013). From baseline to posttreatment, scores decreased in three participants and increased in the other four participants. At follow-up, all five participants who completed the measures (two participants did not complete follow-up
Table 1 Anxiety, depression, and cardiac-related quality of life

\begin{tabular}{lccc}
\hline & Baseline & Post treatment & Follow-up \\
\hline Anxiety (STAI-Y1) & & \\
Mean & 50.83 & 51.71 & 42.6 \\
SD & 10.25 & 8.62 & 8.26 \\
Scale range & $20-80$ & & \\
Depression (PHQ-9) & & \\
Mean & 8.28 & 9.43 & 6.3 \\
SD & 3.04 & 4.28 & \\
Scale range & $0-27$ & & \\
Cardiac-related quality of life (SAQ-7) & \\
Mean & 77.78 & 71.62 & 11.98 \\
SD & 7.18 & 6.21 & \\
Scale range & $0-100$ & & \\
\hline
\end{tabular}

measures), scored lower on the anxiety measure than their baseline score. For two participants, their anxiety scores remained above the clinical threshold, while three participants had follow-up scores below the suggested indicator of clinically significant anxiety. From baseline to follow-up, the mean score for anxiety decreased by eight points from 50.83 to 42.60 , and the reduction in anxiety scores was the same when considering only participants who completed both baseline and follow-up measures.

Three participants had scores suggestive of clinically significant depressive symptoms at baseline and posttreatment (PHQ-9 $\geq 10$; McManus et al., 2005). Two individuals with elevated symptoms at posttreatment reported increased symptoms from baseline to posttreatment. At follow-up 3 months later, 1 of the 5 participants had a score that continued to exceed the cut-point for clinical severity. The mean from baseline to follow-up decreased by two points from 8.28 to 6.30 .

For cardiac-related quality of life, nearly all subscale and summary scores across time points were in the good or excellent ranges ( $>50$ and $>75$, respectively; Chan et al., 2014). On the summary score, one participant's score decreased by 21 points from baseline to follow-up, though her score continued to be classified in the "good" range. Another participant's scores increased by 17 points from baseline to follow-up. Other participants had smaller changes over the 3 time points.

\section{Discussion}

To the best of the authors' knowledge, this is the first study to pilot a CBT-based group intervention designed specifically for SCAD survivors. This intervention included various strategies used in CBT, including psychoeducation about SCAD and various psychological disorders, as well as stress 
management strategies and cognitive restructuring. Acceptance and values techniques used in ACT and interpersonal effectiveness skills used in DBT were also incorporated.

Given the existing literature showing depression and anxiety symptoms are common in SCAD survivors, we focused on these two aspects of psychological distress using wellvalidated measures. In addition, we evaluated cardiac-related quality of life. Our sample included a single cohort made up of seven female SCAD survivors who completed the 8 -week group, though only five completed 3-month followup surveys.

In terms of anxiety, initially all but 1 participant reported baseline symptoms above a threshold suggestive of a clinical level of anxiety (STAI $\geq 40$; Bunevicius et al., 2013). Although some participants had an increase in symptoms at posttreatment, all participants who completed measures at follow-up reported less anxiety than at baseline, and scores for 3 out of 5 participants were below the clinical threshold at follow-up. For depression, 1 person demonstrated a clinically meaningful decrease of at least 5 points in symptoms from baseline to follow-up (Löwe et al., 2004), and 2 others had a decrease of 4 points at their final instance of measurement. For cardiac-related quality of life, all participants reported good or excellent quality of life across time points.

Though some individuals reported decreased depressive symptoms, improvement across participants seemed to be more consistent on the measure of anxiety. We believe that several factors may contribute to this finding. One is the initial level of distress. All but 1 participant reported a clinical level of severity for anxiety at baseline. In contrast, on the measure of depression, 4 of the 7 participants indicated subthreshold depressive symptoms at baseline. Second, other mechanisms may have contributed to the improvement in anxiety symptoms. Given that research on SCAD remains limited, education about SCAD and about the likelihood of recurrence may have quelled worries about future events. Participants learned relaxation exercises, which have shown to be efficacious at reducing anxiety symptoms (Manzoni et al., 2008). The group discussion also allowed participants an opportunity to approach their distress about their SCAD, share their experiences, receive live feedback from peers, and listen to others' experiences (Edwards et al., 2017). These interactions may have allowed for processing difficult memories of the SCAD event and their concerns about coping and the future. A review of group psychotherapy in cancer patients suggested that avoidance is related to poorer coping whereas emotional expression aids in coping with the condition (Blake-Mortimer et al., 1999). Group discussion may have offered a similar venue for SCAD patients to express their fears and concerns in a supportive context.

Another pattern that was similar across participants was a small increase in symptoms from baseline to posttreatment, followed by a decrease in symptoms at follow-up to below baseline levels. Although most of the posttreatment increases did not rise to the level of clinical significance, it remains worth noting. Given there were only 8 weekly sessions, it is possible that participants needed more time to apply the skills learned and to integrate the group discussions for changes to be reflected in their scores. Some prior intervention studies have similarly demonstrated larger gains at a follow-up time point compared with posttreatment (Luoma et al., 2012).

We believe that it is important that this intervention was delivered in a group format. Other SCAD researchers have suggested that SCAD patients may experience distress, in part, due to being relatively young women who may feel different or socially isolated from others as the result of having experienced a heart attack (Liang et al., 2014). Given the relative rarity of SCAD, survivors are unlikely to meet others who have also experienced a SCAD. Some patients use online forums, such as SCAD-specific Facebook groups, to connect with other survivors. Group treatment by nature affords the opportunity for individuals to learn from each other about coping, as well as to experience a sense of community and feel understood by others with similar experiences. Based on existing research, it appears symptoms of depression and anxiety are often present years after the initial SCAD event (Johnson et al., 2020), as was true in our group. Having participants from different lengths of time post-SCAD may offer opportunities for additional learning that would not be possible in a group of participants who were in similar temporal distance from their initial event. Importantly, these group meetings occurred in a moderated environment with a leader who helped participants process their fears and managed emotional content that was raised by group members.

\section{Limitations}

This study had a number of limitations. First, as a pilot study in a relatively rare illness population, our sample size was small and conducted at a single site. Additionally, we had 1 participant drop out of the group and 2 who were lost to follow-up. We also did not have a control group with which to compare our findings, so causal inferences about symptom remittance are not possible. Our sample was fairly homogeneous in education and socioeconomic status, potentially limiting generalizability to other patient samples. Given these limitations, we put forward our findings tentatively and as a call for further intervention development and investigation.

\section{Future Directions}

Further research in understanding this unique patient population, the psychosocial sequalae from SCAD, and 
interventions to support their coping and adjustment are warranted. More information is needed about the natural course of psychological symptoms post-SCAD and optimal time point(s) for intervention and future studies should include other measures of emotional distress such as insomnia and posttraumatic stress (Johnson et al., 2020) as well as psychosocial constructs like health-related locus of control. A small study of SCAD survivors suggested that their profile of health-related locus of control, including sense of control over their condition from self, chance, doctors, or others, was more similar to cancer patients than to other samples of cardiac patients (Edwards et al., 2019). Understanding more about health-locus of control and its relation to symptoms of distress may help to develop and refine appropriate interventions. Future intervention studies with larger sample sizes are needed to determine whether a CBT-based group intervention may be efficacious in reducing distress. It will be important to identify which aspects of CBT interventions may be most helpful for reducing symptoms of anxiety versus depressive symptoms. In addition, it is equally important to determine which patients are not likely to benefit from intervention given that a minority of patients experienced an exacerbation of symptoms. Possible patient factors to consider are the initial baseline distress, age, time since the SCAD event, mental health history, and other demographic variables.

Finally, given the relative rarity of the condition and thus the difficulty of gathering an adequate number of patients in one physical settings, it is worth developing and investigating creative ways that technology may be used to implement treatment and bring patients together to share their experiences. Cancer support groups have been shown to benefit patients with a variety of psychosocial issues (Gottlieb \& Wachala, 2007). A prior study of a multidisciplinary cardiac rehabilitation program for SCAD survivors also emphasized the importance of a peer support element in their intervention, given that patients are unlikely to know another person who has experienced SCAD except through participating in online SCAD forums (Chou et al., 2016). Although individual interventions also warrant development, we believe group interventions that provide opportunities for SCAD survivors to meet and interact hold particular promise in supporting their coping with a unique condition.

\section{Considerations for Future Studies and Interventions}

One challenge for future research (and to clinical intervention) was identifying an adequately sized group. The relative rarity of SCAD limits the number of eligible participants for such a group, particularly when looking within a single health care system or geographic area. This factor is compounded by the challenges of recruiting for any group intervention-selecting those who are appropriate for the intervention, interested in participating, available at the specified time of the group, and can travel to the clinic weekly throughout the intervention. Offering the group via telehealth would likely reduce some of these barriers, such as including patients in another part of one's state. With the COVID-19 pandemic, many behavioral health providers have gained experience in providing care by telehealth and healthcare systems have updated procedures and policies to support and streamline this model of care. Although research on groups delivered via telehealth is still limited, studies suggest that telehealth groups have similar outcomes to inperson groups (Gentry et al., 2019), and they have been used to reach patients living in rural areas (Morland et al., 2010). Participants in our sample tended to be well-educated and with higher household income levels, which helps in accessing care. Delivering a group intervention via telehealth may improve access to SCAD survivors living in rural areas and those facing other practical barriers, such as transportation or other obligations.

\section{Conclusion}

Emotional distress is common after SCAD. The event is unexpected, the cause is unknown, there is a paucity of treatments, and recurrence occurs in nearly $20 \%$ of patients and is associated with major adverse cardiac events. To our knowledge, this is the first investigation of a psychosocial intervention for SCAD survivors. The findings of this pilot study suggest that a group CBT-based intervention may be beneficial for SCAD survivors experiencing psychological distress, particularly anxiety. Future research is needed to develop and evaluate psychosocial interventions for SCAD survivors who experience elevated psychological distress post-SCAD.

Author Contributions JT contributed to the conception of the intervention and referred SCAD patients at a large academic medical center. KSE contributed to the conception and design of the intervention and study. KSE implemented the intervention and conducted data collection. KCV collected follow-up data. KCV entered and organized the data. KCV and KSE interpreted data. KCV and KSE wrote sections of the first draft. All authors contributed to manuscript revision, read, and approved the submitted version.

Funding The authors received no funding towards this study.

\section{Declarations}

Conflict of interest Kelsey C. Vaca and Jennifer A. Tremmel have no relevant financial or non-financial interests to disclose. Katharine S. Edwards is a member of the medical advisory board for the SCAD Alliance, a nonprofit patient advocacy organization. 
Ethical Approval The methodology for this study was approved by the IRB for Stanford University.

Informed Consent Informed consent was obtained from all individual participants included in the study.

Human and Animal Rights This study was reviewed and approved by the Stanford University Institutional Review Board and performed in accordance with the ethical standards as laid down in the 1964 Declaration of Helsinki and its later amendments.

\section{References}

Barnes, L. L., Harp, D., \& Jung, W. S. (2002). Reliability generalization of scores on the spielberger state-trait anxiety inventory. Educational and Psychological Measurement, 62, 603-618. https:// doi.org/10.1177/0013164402062004005

Blake-Mortimer, J., Gore-Felton, C., Kimerling, R., Turner-Cobb, J. M., \& Spiegel, D. (1999). Improving the quality and quantity of life among patients with cancer: A review of the effectiveness of group psychotherapy. European Journal of Cancer, 35, 15811586. https://doi.org/10.1016/S0959-8049(99)00194-X

Blumenthal, J. A., Sherwood, A., \& Babyak, M. A. (2005). Effects of exercise and stress management training on markers of cardiovascular risk in patients with ischemic heart disease: A randomized controlled trial. ACC Current Journal Review, 14, 2-3. https://doi. org/10.1016/j.accreview.2005.06.008

Blumenthal, J. A., Sherwood, A., Smith, P. J., Watkins, L., Mabe, S., Kraus, W. E., Ingle, K., Miller, P., \& Hinderliter, A. (2016). Enhancing cardiac rehabilitation with stress management training: A randomized, clinical efficacy trial. Circulation, 133, 13411350. https://doi.org/10.1161/CIRCULATIONAHA.115.018926

Bunevicius, A., Staniute, M., Brozaitiene, J., Pop, V. J., Neverauskas, J., \& Bunevicius, R. (2013). Screening for anxiety disorders in patients with coronary artery disease. Health and Quality of Life Outcomes, 11, 37. https://doi.org/10.1186/1477-7525-11-37

Chan, P. S., Jones, P. G., Arnold, S. A., \& Spertus, J. A. (2014). Development and validation of a short version of the Seattle Angina questionnaire. Circulation, 7, 640-647. https://doi.org/10.1161/ CIRCOUTCOMES.114.000967

Chou, A. Y., Prakash, R., Rajala, J., Birnie, T., Isserow, S., Taylor, C. M., Ignaszewski, A., Chan, S., Starovoytov, A., \& Saw, J. (2016). The first dedicated cardiac rehabilitation program for patients with spontaneous coronary artery dissection: Description and initial results. Canadian Journal of Cardiology, 32, 554-560. https:// doi.org/10.1016/j.cjca.2016.01.009

Edwards, K. S., Clemson, K. E., \& Tremmel, J. A. (2017). Psychosocial treatment for patients with spontaneous coronary artery dissection. Paper presented at the 38th Annual Meeting and Scientific Sessions of the Society of Behavioral Medicine, San Diego, CA.

Edwards, K. S., Vaca, K. C., Naderi, S., \& Tremmel, J. A. (2019). Patient-reported psychological distress after spontaneous coronary artery dissection: Evidence for post-traumatic stress. Journal of Cardiopulmonary Rehabilitation and Prevention, 39, E20-E23. https://doi.org/10.1097/HCR.0000000000000460

Elderon, L., Smolderen, K. G., Na, B., \& Whooley, M. A. (2011). Accuracy and prognostic value of American Heart Associationrecommended depression screening in patients with coronary heart disease: Data from the heart and soul study. Circulation, 4, 533-540. https://doi.org/10.1161/CIRCOUTCOMES.110.960302
Gentry, M. T., Lapid, M. I., Clark, M. M., \& Rummans, T. A. (2019). Evidence for telehealth group-based treatment: A systematic review. Journal of Telemedicine and Telecare, 25, 327-342.

Gilbody, S., Richards, D., Brealey, S., \& Hewitt, C. (2007). Screening for depression in medical settings with the patient health questionnaire (PHQ): A diagnostic meta-analysis. Journal of General Internal Medicine, 22, 1596-1602.

Gottlieb, B. H., \& Wachala, E. D. (2007). Cancer support groups: A critical review of empirical studies. Psycho-Oncology, 16, 379400. https://doi.org/10.1002/pon.1078

Hayes, S. N., Kim, E. S. H., Saw, J., Adlam, D., Arslanian-Engoren, C., Economy, K. E., Ganesh, S. K., Gulati, R., Lindsay, M. E., Mieres, J. H., Naderi, S., Shah, S., Thaler, D. E., Tweet, M. S., \& Wood, M. J. (2018). Spontaneous coronary artery dissection: Current state of the science: A scientific statement from the American Heart Association. Circulation. https://doi.org/10.1161/CIR. 0000000000000564

Johnson, A. K., Hayes, S. N., Sawchuk, C., Johnson, M. P., Best, P. J., Gulati, R., \& Tweet, M. S. (2020). Analysis of posttraumatic stress disorder, depression, anxiety, and resiliency within the unique population of spontaneous coronary artery dissection survivors. Journal of the American Heart Association, 9, e014372. https:// doi.org/10.1161/JAHA.119.014372

Kim, E. S. H. (2020). Spontaneous coronary-artery dissection. New England Journal of Medicine, 383, 2358-2370. https://doi.org/ 10.1056/NEJMra2001524

Kroenke, K. (2012). Enhancing the clinical utility of depression screening. CMAJ, 184, 281-282. https://doi.org/10.1503/cmaj.112004

Kroenke, K., Spitzer, R. L., \& Williams, J. B. W. (2001). The PHQ-9: Validity of a brief depression severity measure. Journal of General Internal Medicine, 16, 606-613. https://doi.org/10.1046/j. 1525-1497.2001.016009606.x

Liang, J. J., Tweet, M. S., Hayes, S. E., Gulati, R., \& Hayes, S. N. (2014). Prevalence and predictors of depression and anxiety among survivors of myocardial infarction due to spontaneous coronary artery dissection. Journal of Cardiopulmonary Rehabilitation and Prevention, 34, 138-142. https://doi.org/10.1097/ HCR.0000000000000030

Lichtman, J. H., Bigger, J. T., Blumenthal, J. A., Frasure-Smith, N., Kaufmann, P. G., Lesperance, F., Mark, D. B., Sheps, D. S., Taylor, C. B., \& Froelicher, E. S. (2008). Depression and coronary heart disease: Recommendations for screening, referral, and treatment: A science advisory from the American Heart Association prevention committee of the council on cardiovascular nursing, council on clinical cardiology, council on epidemiology and prevention, and interdisciplinary council on quality of care and outcomes research: Endorsed by the American Psychiatric Association. Circulation, 118, 1768-1775. https://doi.org/10.1161/ CIRCULATIONAHA.108.190769

Löwe, B., Unützer, J., Callahan, C. M., Perkins, A. J., \& Kroenke, K. (2004). Monitoring depression treatment outcomes with the patient health questionnaire-9. Medical Care, 42, 1194-1201.

Luoma, J. B., Kohlenberg, B. S., Hayes, S. C., \& Fletcher, L. (2012). Slow and steady wins the race: A randomized clinical trial of acceptance and commitment therapy targeting shame in substance use disorders. Journal of Consulting and Clinical Psychology, 80, 43-53. https://doi.org/10.1037/a0026070

Manzoni, G. M., Pagnini, F., Castelnuovo, G., \& Molinari, E. (2008). Relaxation training for anxiety: A ten-years systematic review with meta-analysis. BMC Psychiatry, 8, 41. https://doi.org/10. 1186/1471-244X-8-41

McManus, D., Pipkin, S. S., \& Whooley, M. A. (2005). Screening for depression in patients with coronary heart disease (data from the heart and soul study). The American Journal of Cardiology, 96, 1076-1081. https://doi.org/10.1016/j.amjcard.2005.06.037 
Morland, L. A., Greene, C. J., Rosen, C. S., Foy, D., Reilly, P., Shore, J., He, Q., \& Frueh, B. C. (2010). Telemedicine for anger management therapy in a rural population of combat veterans with posttraumatic stress disorder: A randomized noninferiority trial. The Journal of Clinical Psychiatry, 71, 855-863.

Orth-Gomér, K., Schneiderman, N., Wang, H.-X., Walldin, C., Blom, M., \& Jernberg, T. (2009). Stress reduction prolongs life in women with coronary disease: The Stockholm women's intervention trial for coronary heart disease (SWITCHD). Circulation, 2, 25-32. https://doi.org/10.1161/CIRCOUTCOMES.108.812859

Saw, J., Humphries, K., Aymong, E., Sedlak, T., Prakash, R., Starovoytov, A., \& Mancini, G. B. J. (2017). Spontaneous coronary artery dissection: Clinical outcomes and risk of recurrence. Journal of the American College of Cardiology, 70, 1148-1158. https://doi. org/10.1016/j.jacc.2017.06.053

Speilberger, C. D., \& Gorsuch, R. L. (1983). Manual for the state-trait anxiety inventory (Form Y) ("self-evaluation questionnaire"). Consulting Psychologists Press.

Spertus, J. A., Winder, J. A., Dewhurst, T. A., Deyo, R. A., Prodzinski, J., McDonnell, M., \& Fihn, S. D. (1995). Development and evaluation of the Seattle Angina questionnaire: A new functional status measure for coronary artery disease. Journal of the American College of Cardiology, 25, 333-341.

Thekkumpurath, P., Walker, J., Butcher, I., Hodges, L., Kleiboer, A., O'Connor, M., Wall, L., Murray, G., Kroenke, K., \& Sharpe, M. (2011). Screening for major depression in cancer outpatients: The diagnostic accuracy of the 9-item patient health questionnaire. Cancer, 117, 218-227. https://doi.org/10.1002/cncr.25514

Wagers, T. P., Stevens, C. J., Ross, K. V., Leon, K. K., \& Masters, K. S. (2018). Spontaneous coronary artery dissection (SCAD): Female survivors' experiences of stress and support. Journal of Cardiopulmonary Rehabilitation and Prevention, 38, 374-379. https://doi.org/10.1097/HCR.0000000000000330

Publisher's Note Springer Nature remains neutral with regard to jurisdictional claims in published maps and institutional affiliations. 